\title{
Editorial: Natural Compounds in Food Safety and Preservation
}

\author{
Susana Ferreira ${ }^{1 *}$, Gloria Sanchez ${ }^{2}$, Maria J. Alves ${ }^{3}$ and Maria J. Fraqueza ${ }^{4}$ \\ ${ }^{1}$ CICS-UBI - Health Sciences Research Centre, University of Beira Interior, Covilhã, Portugal, ${ }^{2}$ Department of Preservation \\ and Food Safety Technologies, Instituto de Agroquímica y Tecnología de Alimentos - Consejo Superior de Investigaciones \\ Científicas (IATA-CSIC), Valencia, Spain, ${ }^{3}$ Centro de Investigação de Montanha (CIMO), Instituto Politécnico de Bragança, \\ Bragança, Portugal, ${ }^{4}$ CIISA-Centro de Investigação Interdisciplinar em Sanidade Animal, Faculdade de Medicina Veterinária, \\ Universidade de Lisboa, Lisboa, Portugal
}

Keywords: editorial, natural compounds, food safety, food preservation, antimicrobial

\section{Editorial on the Research Topic}

\section{Natural Compounds in Food Safety and Preservation}

Food safety is a global challenge, with foodborne diseases posed as a relevant concern for human health, and food microbial spoilage being a problem for agri-food companies (1). Considerable research has been dedicated to diverse approaches that can be applied to control foodborne pathogens and microbial spoilage, among which the potential use of natural compounds has been highlighted as a strategy for improving food safety, but also quality and extending selflife (1-3). Furthermore, the negative consumer perception of the synthetic preservatives used in food industry, associated with an increasing demand for maintenance of nutritional and quality properties, has encouraged the pursue for the use of natural-based preservatives in food production (1-3).

OPEN ACCESS

Edited and reviewed by: Elena Ibañez, Institute of Food Science Research (CIAL), Spain

*Correspondence:

Susana Ferreira susana.ferreira@fcsaude.ubi.pt

Specialty section: This article was submitted to Nutrition and Food Science Technology, a section of the journal Frontiers in Nutrition

Received: 16 August 2021 Accepted: 23 August 2021 Published: 16 September 2021

Citation:

Ferreira S, Sanchez G, Alves MJ and Fraqueza MJ (2021) Editorial: Natural Compounds in Food Safety and Preservation. Front. Nutr. 8:759594. doi: 10.3389/fnut.2021.759594
In this context, in this Research Topic, natural antimicrobial compounds have been highlighted by their activity against Chronobacter spp. in infant powdered formula by Yemiș and Delaquis. The authors reviewed the potential of natural compounds from plants, microbial and animal sources as alternatives to synthetic chemical preservatives, addressing nutritional, toxicological, and regulatory issues. In fact, the use of natural antimicrobial compounds needs to be guided considering the regulatory framework, and so the authors suggest the use of well-studied single compounds over multiple-component preparations.

Among the natural compounds, essential oils have been pointed as promising antimicrobial mixtures. Yousefi et al. reviewed the potential application of essential oils with antilisterial activity in meat and poultry products, since contaminated meat products are recognized as one main source for Listeria monocytogenes infection. The authors described the efficiency of several essential oils in the control of L. monocytogenes, whilst addressing the mechanism of action of some selected compounds and the major drawbacks associated with the application of essential oils in food products. The activity of natural compounds in food is dependent of various factors, namely on the complexity and composition of the product, this highlights the need of the validation of antimicrobial activity in food matrixes. Kiprotich et al. explored the use of thyme oil combined with Yucca schidigera extract to marinate raw chicken breast meat in lemon juice. The authors considered the potential of antimicrobial marinade formulations as an approach to reduce enteric pathogens. Based on their results, thyme oil showed to be an enhancer of the inactivation of Salmonella enterica on raw chicken breast, increasing the antimicrobial efficacy of lemon juice marinade containing yucca extract to emulsify the thyme oil.

Besides, the perspective of the use of natural antimicrobial compounds for controlling foodborne pathogens, research has also been carried out to elucidate its use against microbial spoilage. 
Shen et al. reported the antifungal activity of Loquat leaves extract against citrus postharvest pathogens, providing a mechanistic overview of the anti-Penicillium digitatum activity. The antifungal activity of this extract against $P$. digitatum was attributed to the derangement of cell membrane permeability and the disordered energy metabolism.

Among the limitations of the use of essential oils are: offflavors and odors that may result in an unacceptance of the food product by the consumer usually associated with the use of high concentrations of essential oils, the degradation of the components or the limited interaction with the microorganisms. The incorporation of these natural bioactive compounds, into edible coatings, food packaging materials, or other formulations may be presented as an approach to overcome these problems. Asensio et al. reported the use of nanoemulsions as an approach to encapsulate, protect, and deliver Argentinean oregano essential oil. The authors optimized the physical stability of the nanoemulsion and characterized it, showing that the formulation may even increase the antimicrobial activity and inhibition of quorum sensing when comparing with the pure essential oil.

The application of these natural compounds may be accomplished alone or in combination with already existing preservatives or even processing methods for the development

\section{REFERENCES}

1. Chibane LB, Degraeve P, Ferhout H, Bouajila J, Oulahal N. Plant antimicrobial polyphenols as potential natural food preservatives. J Sci Food Agric. (2019) 99:1457-74. doi: 10.1002/jsfa.9357

2. Tiwari BK, Valdramidis VP, O’Donnell CP, Muthukumarappan K, Bourke P, Cullen PJ. Application of natural antimicrobials for food preservation. J Agric Food Chem. (2009) 57:5987-6000. doi: 10.1021/jf900668n

3. Calo JR, Crandall PG, O’Bryan CA, Ricke SC. Essential oils as antimicrobials in food systems - a review. Food Control. (2015) 54:111-9. doi: 10.1016/j.foodcont.2014.12.040

Conflict of Interest: The authors declare that the research was conducted in the absence of any commercial or financial relationships that could be construed as a potential conflict of interest. of a food preservation system, providing mechanisms to ensure food safety. This subject was approached by Barroug et al. who reviewed the use of natural compounds with non-thermal strategies on poultry products addressing the effects on the microbiological and physicochemical characteristics. This paper gives a well-balanced overview of the use of non-thermal technologies, natural compounds and their combination as an intervention for safer poultry products.

In conclusion, the present Research Topic provides several examples of natural antimicrobial compounds and their application in different contexts.

\section{AUTHOR CONTRIBUTIONS}

SF drafted the manuscript. GS, MA, and MF provided critical review and insight and revised the final version of the editorial. All authors contributed to the article and approved the submitted version.

\section{ACKNOWLEDGMENTS}

The editors of this topic would like to thank all authors and reviewers for their contributions to the present collection.

Publisher's Note: All claims expressed in this article are solely those of the authors and do not necessarily represent those of their affiliated organizations, or those of the publisher, the editors and the reviewers. Any product that may be evaluated in this article, or claim that may be made by its manufacturer, is not guaranteed or endorsed by the publisher.

Copyright (c) 2021 Ferreira, Sanchez, Alves and Fraqueza. This is an open-access article distributed under the terms of the Creative Commons Attribution License (CC $B Y)$. The use, distribution or reproduction in other forums is permitted, provided the original author(s) and the copyright owner(s) are credited and that the original publication in this journal is cited, in accordance with accepted academic practice. No use, distribution or reproduction is permitted which does not comply with these terms. 